\title{
Pre-sharpened Microtips: An Efficient Sample Preparation Method for Atom Probe
}

\section{Tomography}

R. Shivaraman ${ }^{1}$, R. M. Ulfig ${ }^{1}$, D. J. Larson ${ }^{1}$, H. Fukuzawa ${ }^{2}$, D. C. Bell ${ }^{3}$, H. Wang ${ }^{3}$, R. Gordon ${ }^{3}$

${ }^{1}$ Imago Scientific Instruments Corporation, 5500 Nobel Dr., Madison WI 53711

${ }^{2}$ Corporate Research \& Development Center, Toshiba Corporation, Kawasaki 212-8582, Japan

${ }^{3}$ Center for Nanoscale Systems, Harvard University, 11 Oxford St, Cambridge MA 02138

The principal lens of a Local Electrode Atom Probe (LEAPTM) microscope is the specimen. Hence for accurate and rapid atomic-scale characterization, proper selection of the sample preparation technique is paramount. Imago Scientific Instruments introduced the silicon-based pre-sharpened microtips (PSMs) [1] with a radius of curvature at the apex of $\leq 50 \mathrm{~nm}$ to allow direct deposition of various thin films and en masse production of atom-probe-ready tips. The use of Si PSMs eliminates all post-deposition processing steps, and hence circumvents the damage caused by gallium implantation which occurs during focused-ion-beam milling process [2]. PSMs also enable repeated analysis conducted on the same materials system which assists in validating repeatability and provides valuable feedback that may shorten research and development cycle lifetimes. This work presents a variety of applications of LEAP analysis to multicomponent films deposited on PSMs.

The advancement of data storage technology relies on the ability to engineer, characterize and optimize the nanostructural features present in multicomponent layered structures to achieve desired magnetic properties. The following giant-magnetoresistance (GMR) multilayer stack was deposited by DC magnetron sputtering onto a PSM coupon: buffer $/ \mathrm{PtMn} / \mathrm{CoFe} / \mathrm{Ru} / \mathrm{CoFe} / \mathrm{Cu}-$ $\mathrm{Al}_{2} \mathrm{O}_{3} / \mathrm{CoFe} / \mathrm{NiFe} / \mathrm{cap}$ [3]. The goal was to quantify $\mathrm{Cu}$ segregation in the nano-oxide layer (NOL) and determine the alignment of the current-confined-path (CCP) with the grains in the magnetic layers. Fig. 1 shows $50 \% \mathrm{Cu}$ isoconcentration surfaces in $\mathrm{CCP}-\mathrm{NOL}$ and $77 \% \mathrm{Ni}$ surfaces in the $\mathrm{NiFe}$ layer. The 3-D reconstructions indicate the formation of CCP regions near the middle of the $\mathrm{NiFe}$ grains of the magnetic free layer [3]. This alignment results in increased GMR because of the reduced possibility of diffusive scattering of conduction electrons at the grain boundaries.

Fig. 2 shows a reconstruction of $\mathrm{WN} / \mathrm{Ru}\left(\mathrm{NH}_{3}\right) / \mathrm{Ru}\left(\mathrm{O}_{2}\right)$ films deposited on a PSM via atomic layer deposition. These films act as a diffusion barrier for interconnect copper metallization. The morphology, resistivity \& adhesion of the Ru layer are governed by its impurity content. LEAP analysis was employed to quantify the various impurities present in these films. The mass spectrum shown in Fig. 3 obtained from the specimen displays a mass resolving power of $\sim 1100$ (FWHM) and $\sim 560$ (FWTM) with negligible background noise.

It is also of interest to quantify the effects of the PSM curvature on the morphology of the deposited thin films. A CoCrPt-based alloy used in perpendicular magnetic recording media [4] was deposited (with a Ru seed layer) on PSMs by magnetron sputtering. The grain structure and crystalline orientation of the CoCrPt phase depends on those of the underlying Ru layer. A reconstruction of the $\mathrm{Ru}$ layer shown in Fig. 4 clearly delineates the grain morphology (using isodensity surfaces at $30 \%$ $\mathrm{Ru}$ ). Spatial Distribution Maps ${ }^{\mathrm{TM}}$ (SDM) [5] obtained from different Ru grains give an interplanar spacing of $\sim 2 \AA$ which is consistent with (0002) planes of hcp-Ru and shows that high quality film deposition on PSMs is achievable. PSMs are the specimen preparation method of choice for accurate and timely $3 \mathrm{D}$-characterization of a wide spectrum of multilayer film structures.

[1] K. Thompson et al. Microsc. Microanal. 11 (S2) (2005) 882.

[2] D. J. Larson et al. Ultramicroscopy 79 (1999) 287. 
[3] H. Fukuzawa et al. IEEE Trans. Mag. 44(11) (2008) 3580.

[4] T. Oikawa et al. IEEE Trans. Mag. 38(5) (2002) 1976.

[5] B. P. Geiser et al. Microsc. Microanal. 13(6) (2007) 437.

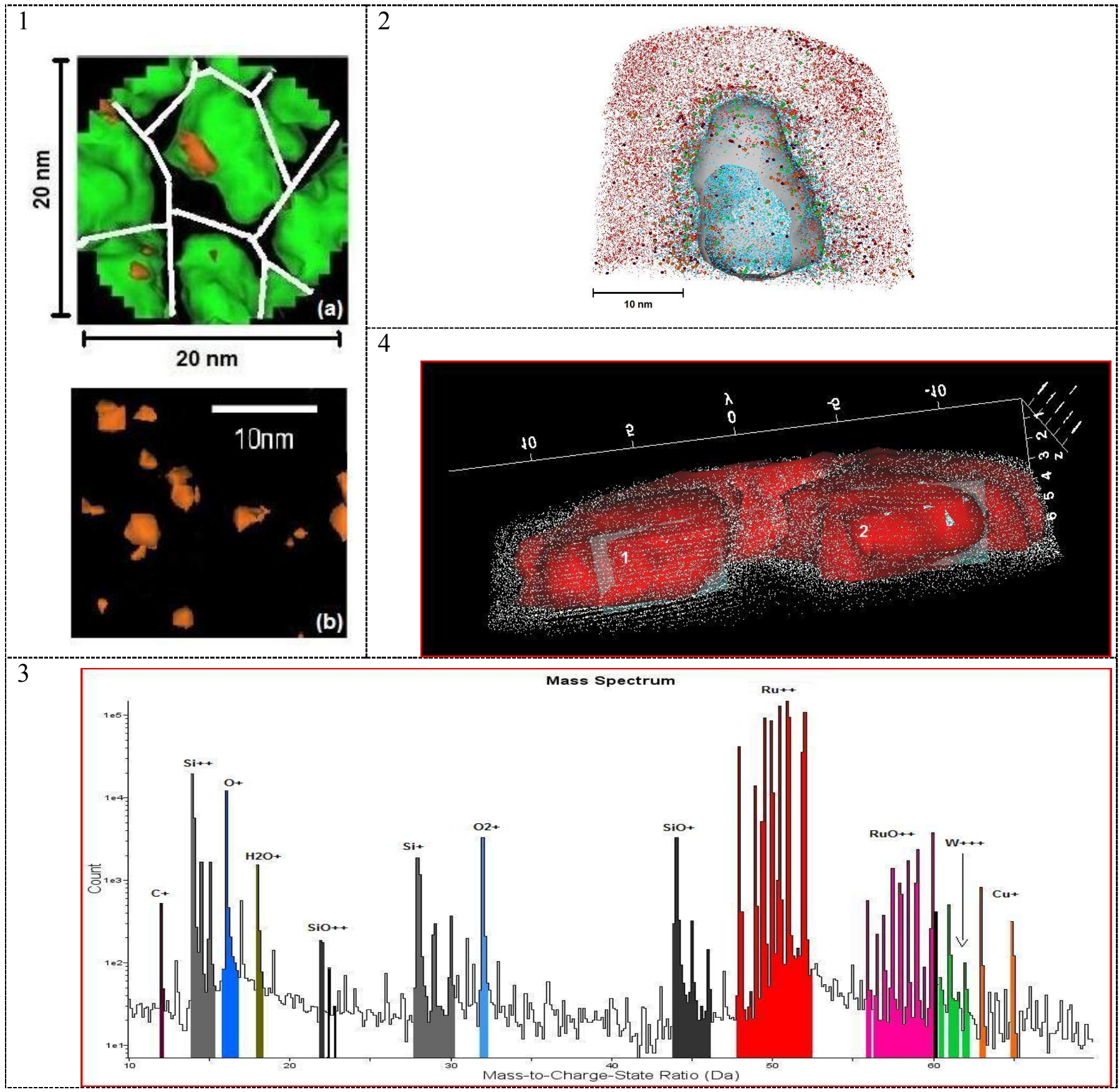

Fig. 1. (a) LEAP reconstruction of $50 \% \mathrm{Cu}$ (orange) and $77 \% \mathrm{Ni}$ (green) isoconcentration surfaces in a current-confined-path nano-oxide layer (b) $\mathrm{Cu}$ segregation only (from ref. [3]).

Fig. 2. $\mathrm{Si}(20$ at. \%) isosurface used to illustrate PSM shape. Atom map includes Ru (red), W (green), O (blue) \& C (brown).

Fig. 3. Mass Spectrum from WN/Ru layers (Fig. 2) deposited on Silicon PSM.

Fig. 4. Grain morphology of Ru underlayer. SDMs were obtained from grains $1 \& 2$. 